\title{
Catalogue $^{\star}$ of HII regions in NGC 7331 at the 6 meter telescope
}

\author{
H. Petit \\ Observatoire de Marseille, 2 place Le Verrier, F-13248 Marseille Cedex 04, France
}

Received February 5; accepted March 19, 1998

\begin{abstract}
A catalogue of HII regions in NGC 7331 is presented, with positions relative to the center of the Galaxy and relative $\mathrm{H} \alpha$ fluxes. The catalogue can be found as an electronic file at the CDS related to the companion paper "Catalogue of HII region measured on 6 meter telescope plates at Observatoire de Marseille".
\end{abstract}

Key words: galaxies: NGC 7331 - galaxies: ISM HII regions - catalogue

Several years ago, a program of $\mathrm{H} \alpha$ narrow-band photography of HII regions in galaxies was undertaken with the $\mathrm{f} / 1$ focal reducer of Courtès attached at the prime focus of the 6-m telescope of the soviet Special Astrophysical Observatory in Zelentchuk. In this catalogue we present the results on the galaxy NGC 7331 .

The photograph was obtained at the prime focus reducer of Courtès (Courtès et al. 1987). It is a $120 \mathrm{~m}$ exposure on a 4415 Kodak film. The $20^{\prime}$ circular field encompasses the full galaxy. The angular resolution is limited by the seeing to about $1^{\prime \prime} .5$. The interference filter is centered at $6557 \AA$ and is $25 \AA$ wide (FWHM).

A preliminary identification of the HII regions was conducted by visual intercomparison of the $\mathrm{H} \alpha$ and continuum photograph. It permitted to reject foreground stars and gelatine defects. The $\mathrm{H} \alpha$ photograph was then digitized with the MAMA microdensitometer of the Observatoire de Paris using a 10 microns square scanning slit and a 10 microns sampling step. The data reduction was performed on the VAX 4000/500 computer of the Observatoire de Marseille using MIDAS and special procedures designed within the framework of MIDAS. The method is the same than that used for M 81 (Petit et al. 1988), NGC 2403 (Sivan et al. 1990) and M 51 (Petit et al. 1996). The photographic densities were converted into ar-

Send offprint requests to: $\mathrm{H}$. Petit

* The catalogue is available at the CDS via anonymous ftp 130.79.128.5 or http://cdsweb.u-strasbg.fr/Abstract.html bitrary intensities using a relative photometric calibration of the Kodak emulsion.

We assigned to each HII region the $X, Y$ positions of the photometric centre provided by the corresponding isophotal system. The $X, Y$ coordinates, measured on the MAMA reference frame, were then converted into a system centred on the nucleus of NGC 7331, with the $X$ and $Y$ axes in the $\mathrm{E}-\mathrm{W}$ and $\mathrm{N}-\mathrm{S}$ directions respectively.

The flux for each HII region was computed by interactive integration of the intensity contained within the outermost isophote down to the local threshold and corrected for the background intensity; the aperture used for the $\mathrm{H} \alpha$ flux integration of the individual HII regions corresponds to $20^{\prime \prime}$ in diameter.

The diameters of the HII regions were measured following the method used in previous papers (Milliard \& Marcelin 1981; Petit et al. 1988; Sivan et al. 1990; Courtès et al. 1993; Petit et al. 1996). These authors define the effective diameter of the HII region as the diameter of the region measured at half its total flux, corrected for instrumental broadening.

The correction is made by comparison with the observed half-flux diameter of the field star images which have the same integrated flux. In other words, the effective diameter is given by $D=2 k\left(A 2-A 2^{*}\right)^{1 / 2} \sqrt{\pi}$ where $k$ is a scale factor, and $A 2$ and $A 2^{*}$ are the areas on the image containing half the total flux, for the HII region and for a field star of the same total flux, respectively. This method allowed us to determine the effective diameters for $55.6 \%$ of the identified HII regions $\left(A 2>A 2^{*}\right)$. The half-flux effective diameter method is well adapted to the HII regions of relatively homogeneous brightnesses, which is, for obvious reasons, the case for HII region diameters close to the seeing disk (for distant galaxies this case is the most frequent).

The table gives the following information:

Column 1: name of the galaxy.

Column 2: lists the reference number given to the individual HII regions. 
Columns 3 and 4: give the $\alpha \delta$ position (1950) of the centre of the galaxy.

Columns 5 and 6 : give the $X, Y$ positions of the photometric centre of the regions.

Column \%: lists the total $\mathrm{H} \alpha$ fluxes of the regions in arbitrary units.

Column 8: gives the effective (half-flux) diameter $D$ in arcsec.

Column 9: gives the geometrical diameter in arcsec.

\section{Conclusion}

We have identified a total of 252 HII regions in NGC $7331 ; 44 \%$ of them are diffuse ones. The data confirm some general properties previously found in a great number of spiral galaxies; the distribution of diameters is well represented by the van den Bergh's (1981) law.
These data will be compared to a model in a very next future to study the spiral arms structure.

The data are included in the Catalogue of HII regions at the $6 \mathrm{~m}$ telescope (Petit 1998).

\section{References}

Courtès G., Petit H., Sivan J.P., Dodonov S., Petit M., 1987, A\&A 174, 28

Courtès G., Petit H., Hua C.T., et al., 1993, A\&A 268, 419

Milliard B., Marcelin M., 1981, A\&A 95, 59

Petit H., 1998, A\&AS 131

Petit H., Sivan J.P., Karachentsev I.D., 1988, A\&A 74, 475

Petit H., Hua C.T., Bersier D., Courtès G., 1996, A\&A 309, 446

Sivan J.P., Petit H., Comte G., Maucherat A.J., 1990, A\&A 237, 23

Van der Bergh S., 1981, AJ 86, 1464 